Article

\title{
Clausius' Disgregation: A Conceptual Relic that Sheds Light on the Second Law
}

Emilio Marco Pellegrino ${ }^{1}$, Elena Ghibaudi ${ }^{1, *}$ and Luigi Cerruti ${ }^{2}$

1 Department of Chemistry, University of Torino, Via Giuria 7, I-10125, Torino, Italy;

E-Mail: emiliomarco.pellegrino@unito.it

2 Independent researcher, Viale delle acacie 12, I-10148, Vinovo, Italy;

E-Mail: 1cerruti00@gmail.com

* Author to whom correspondence should be addressed; E-Mail: elena.ghibaudi@unito.it;

Tel.: +39-11-670-7951 (ext. 123); Fax: +39-11-670-7855.

Academic Editor: Kevin H. Knuth

Received: 5 May 2015 / Accepted: 17 June 2015 / Published: 25 June 2015

\begin{abstract}
The present work analyzes the cognitive process that led Clausius towards the translation of the Second Law of Thermodynamics into mathematical expressions. We show that Clausius' original formal expression of the Second Law was achieved by making extensive use of the concept of disgregation, a quantity which has subsequently disappeared from the thermodynamic language. Our analysis demonstrates that disgregation stands as a crucial logical step of such process and sheds light on the comprehension of such fundamental relation. The introduction of entropy — which occurred three years after the first formalization of the Second Law — was aimed at making the Second Law exploitable in practical contexts. The reasons for the disappearance of disgregation, as well as of other "pre-modern" quantities, from the thermodynamics language are discussed.
\end{abstract}

Keywords: disgregation; entropy; Second Law; Clausius

PACS Codes: 05.70.-a; 01.70.+w 


\section{Introduction}

Despite the fact that the first formulation of the Second Law is almost 200 years old, its adolescence does not seem to be over yet. The controversial character of this law is still the object of an extended debate, its validity is "under increased scrutiny" and, according to Čápek and Sheehan, since "the early 1980's, roughly 50 papers representing over 20 challenges have appeared in the refereed scientific literature" [1] (p. xiii). Nikulov and Sheehan depict the cultural climate that surrounds the Second Law from this provocative perspective:

“... like most paradigms, the Second Law is understood deeply by few and taken on faith by most. Such faith is cemented by many famous endorsements and is so deeply rooted in a century and a half of cultural legacy that it has put the Second Law nearly beyond the reach of serious scientific discussion. Taken together, these constitute what may be called the Second Law mystique." [2] (p. 1)

Uffink provides another interesting standpoint that may be seen as an enforcement of the foregoing passage:

"The Second Law made its appearance in physics around 1850, but a half century later it was already surrounded by so much confusion that the British Association for the Advancement of Science decided to appoint a special committee with the task of providing clarity about the meaning of this law. However, its final report [3] did not settle the issue. Half a century later, the physicist/philosopher Bridgman still complained that there are almost as many formulations of the Second Law as there have been discussions of it [4] (p. 116). And even today, the Second Law remains so obscure that it continues to attract new efforts at clarification.” [5] (p. 306)

Aims of the present paper are: (i) to describe and analyze the cognitive process that led Clausius to translate the Second Law into mathematical expressions; (ii) to discuss the role played by some "pre-modern" quantities, namely disgregation, in the above-cited process.

Our thesis is that the epistemic role played by these quantities is crucial and that their disappearance does not affect the self-consistency of Clausius' formal system. In particular, we will show that the concept of disgregation stands out as a mandatory step of the pathway that led Clausius towards the formalization of the Second Law.

Our analysis is mainly based on Clausius' Fourth, Sixth, and Ninth Memoirs [6]. We will also refer to an argumentative paper published by Clausius in 1872 with the title Zur Geschichte der mechanischen Wärmetheorie [7]. This paper-written in the frame of Clausius' dispute with the English thermodynamics community [8] (p. 64) - contains Clausius' description of the evolution of his theory up to the definition of entropy.

\section{Historical Background}

Müller points out that entropy first emerged "in the context of the engineering proposition [...] of how to improve the efficiency of heat engines" [8] (p. 47). This holds true for the Second Law as well. At the beginning of the 18th century, England was facing an important energy crisis whose origin can 
be ascribed to the following reasons: (i) England was almost deforested and the remaining trees were needed by the navy; (ii) the output of coal extraction decreased significantly and most mines were under threat of closure as pits were flooded and needed drainage. Newcomen's steam engine [9] was invented in this economical context.

At first, steam machines served mainly as pumps for the drainage of coalmines, as they allowed reaching greater pits' depths and consequently improving coal extraction. Later, the steam engine was also employed by the iron industry for crushing ores and it quickly became a strategic industrial facility. The efficiency of Newcomen's machine was actually very low (about 2\%) [10] so that coal was mostly consumed for heating the engine's boiler. Subsequently, James Watt (1736-1819) improved the efficiency up to $5-7 \%$ by inserting a separate cooler, wherein the steam was pushed before condensation [11]. Furthermore, the efficiency of the engines gradually continued to be improved by engineers and in the 1820 s it had reached $18 \%$. At that time Sadi Carnot started to investigate how far this improvement could possibly go. In the introduction of his book, Carnot depicts the economic landscape of England in a way that does not correspond any longer to the energy crisis scenery of the beginning of the preceding century:

"To take away today from England her steam engines would be to take away at the same time her coal and iron. It would be to dry up all her sources of wealth, to ruin all on which her prosperity depends, in short, to annihilate that colossal power.” [12] (p. 40)

Indeed, Carnot's memoire is a complex paper containing relevant and controversial issues that were the object of several critical reviews. Uffink, in his paper on the Second Law of Thermodynamics, reports an elaborated analysis of Carnot's arguments and — introducing his work- he writes:

"It was this work which eventually led to the birth of thermodynamics. Still, Carnot's memoir itself does not belong to what we presently understand as thermodynamics. It was written from the point of view that heat is an indestructible substance, the so-called calorique. This assumption proved to be in conflict with empirical evidence. However, Carnot's main theorem did agree with experience. Classical thermodynamics was born from the attempts around 1850 to save Carnot's theorem by placing it on a different footing." [5] (p. 320)

Carnot's theorem, in its own author's words, corresponds to the following proposition:

"The motive power of heat is independent of the agents employed to realize it; its quantity is fixed solely by the temperature of the bodies between which is effected, finally the transfer of the calorique." [12] (p. 68)

Sands and Dunning-Davies remark that Clausius proved this theorem to be valid, despite its being grounded on the erroneous assumption of calorique.

"Carnot's theory, though founded on the notion of heat as caloric, survives the abandonment of this theory and the adoption of the dynamical theory of heat. Carnot's theorem that the conversion of heat into work, and vice versa, depends only on the temperatures of the hot and cold reservoirs and not on the nature of the working fluid, was subsequently shown by Clausius to hold true with the adoption of the mechanical theory of heat." [13] (p. 3) 


\section{The Second Law of Clausius' Mechanical Theory of Heat}

\subsection{A Brief Overview of Clausius' Work}

In 1850 Clausius published his first paper on the Mechanical Theory of Heat, wherein he rejected the old theory of calorique, reaffirmed the validity of Carnot's theorem and proposed the first formulation of what will be known as the Second Law of Thermodynamics [6] (pp. 14-69). A few months later, in 1851, W. Thomson published a second formulation of the Second Law, that is basically equivalent to Clausius' one. Both are largely based on the critical analysis of Carnot's work [14] and are expressed as qualitative statements. Clausius' first formulation states that: "the heat cannot by itself flow from a colder body to a warmer one" [7] (p. 136) (translated by the authors).

In the following sections we will describe and analyze how Clausius translated this qualitative statement into a mathematical expression. The reconstruction of this cognitive process is based on Clausius' original works. Clausius' Mechanical Theory of Heat underwent a number of transformations through a logical process that took almost 15 years and is grounded on two premises: (i) Clausius' idea of heat and work, implying a microscopic view of thermodynamic systems; (ii) the use of pre-modern quantities that are no longer in use in current thermodynamics. A preliminary brief presentation of these foundational elements is necessary for the later discussion.

\subsection{Clausius' Idea of Heat and Work}

Clausius' idea of heat is grounded on an atomistic representation of matter wherein heat is associated with the motion of "constituent particles". Such idea is intimately connected to the kinetic model that he developed since 1857 [15] with the aim of providing a microscopic description of heat. According to Brush, Clausius "had been thinking about molecular motions even before writing his first article on thermodynamics in 1850" [16]. On this topic, Clausius himself declared:

"Before writing my first memoir on heat, which was published in 1850, and in which heat is assumed to be a motion, I had already formed for myself a distinct conception of the nature of this motion, and had even employed the same in several investigations and calculations. In my former memoirs I intentionally avoided mentioning this conception, because I wished to separate the conclusions that are deducible from certain general principles from those which presuppose a particular kind of motion, because I hoped to be able at some future time to devote a separate memoir to my notion of this motion and to the special conclusions which flow therefrom." [17] (p. 112)

More specifically, in Clausius' thought, heat was associated to the kinetic energy of particles, called vis viva in 19th century scientific language, as witnessed by the following passage of the First Memoir:

"We shall forbear entering at present on the nature of the motion which may be supposed to exist within a body, and shall assume generally that a motion of the particles does exist, and that heat is the measure of their vis viva.

Or yet more generally, we shall merely lay down one maxim which is founded on the above assumption: In all cases where work is produced by heat, a quantity of heat proportional to 
the work done is consumed; and inversely, by the expenditure of a like quantity of work, the same amount of heat may be produced."[6] (p. 18).

Sands and Dunning-Davies [13] underline that this archaic view of heat permeates all Clausius' writings and is typical of the thinking of that time. According to these authors, this opens the question of the validity of the result of Clausius' theory, as "Modern thermodynamics has rejected the notion of heat upon which Clausius based all his thoughts" [13] (p. 3). Consequently, they wonder whether the use of the entropy concept is still justified, as it seems grounded on false premises. Distinct opinions have been expressed on this matter by other authors. For example, Müller remarks that:

"With all this — by Clausius' work of 1850 — thermodynamics acquired a distinctly modern appearance. His assumptions were quickly confirmed by experimenters, or by reference to older experiments, which Clausius had either not known, or not used.” [8] (p. 63)

From our standpoint, this case is similar to that of Carnot's theory that was based on the false assumption that heat is a conserved quantity; nevertheless the theory provided a number of results, such as Carnot' theorem, that have been proven valid beyond the limits of their theoretical premises.

Based on his kinetic model, Clausius represented the relation between heat and work, pointing out the following distinction:

"The forces which here enter into consideration may be divided into two classes: those which the atoms of a body exert upon each other, and which depend, of course, upon the nature of the body, and those which arise from the foreign influences to which the body may be exposed. According to these two classes of forces which have to be overcome (of which the latter are subjected to essentially different laws), I have divided the work done by heat into interior and exterior work." [6] (p. 112)

So, Clausius split the amount of work "performed by the heat during the change of condition of the body" into two quantities: the interior and the exterior work, denoted by $\mathrm{dI}$ and $\mathrm{dW}$, respectively [6] (p. 225, footnote). The meaning of these quantities is extensively discussed in appendix A of the Sixth Memoir [6] (pp. 250-256). However, it is worth reporting an extract of the 1872 paper, wherein these two quantities are characterized as being either independent or dependent on the path of the transformation. This can be seen as an ante litteram reference to the modern concepts of state function and process function, respectively;

"I distinguish the work into interior and exterior work and the difference marking these two instances is that the former is independent from the path of the transformation while the latter is dependent on it." [7] (p.135) (translated by the authors)

The heat was similarly split into two quantities: $d Q$ - the quantity of heat exchanged with external bodies, and $d H$ - the heat actually contained in the body [18]. Hence, the First Law could be written in terms of these quantities:

$$
d Q+d H+A d I+A d W=0
$$

where A is "the thermal equivalent of a unit of work" [19]. This expression takes a more familiar form after the introduction of the new thermodynamic function $U$ that Clausius mathematically expressed as 
the sum [20]:

$$
d U=d H+A d I
$$

By simple substitution, Equation (1) can be written as

$$
d U+d Q+A d W=0
$$

By using the quantity $d L$ that is "the work, both interior and exterior, performed by the heat in the change [i.e., $d L=d I+d W]$ ” [6] (p. 225), Clausius' First Law becomes:

$$
d Q+d H+A d L=0
$$

In conclusion, Clausius' thermodynamics model is based on four fundamental quantities. Two quantities (i.e., the internal work, I, and the heat actually contained in the body, H) are formal entities without correspondence to any macroscopic physical quantity and disappeared over the years. Conversely, the other two quantities (i.e., the external work, $\mathrm{W}$, and the heat exchanged with the external environment, Q) have a precise macroscopic physical meaning (very close to the concepts of work and heat of modern thermodynamics) and lasted over time.

The impossibility to know the values of I and $\mathrm{H}$ - as well as their lack of a clear physical meaning — is a problem that was already highlighted by Maxwell:

"With respect to our knowledge of the condition of energy within a body, both Rankine and Clausius pretend to know something about it. We certainly know how much goes in and comes out and we know whether at entrance or exit it is in the form of heat or work, but what disguise it assumes when in the privacy of bodies, ... is known only to R., C. and Co.” [21] (p. 222).

Clausius was conscious of the difficulty to exploit these quantities separately so that he remarks "I have thought it advisable to introduce a function which shall represent the sum of these quantities and which I have denoted by U" [6] (p. 225, footnote). The physical meaning of U is clarified by the First Law (i.e., it is the algebraic sum of $\mathrm{Q}$ and $\mathrm{W}$ ) and is very close to the concept of internal energy of modern thermodynamics. The formal nature of I and $\mathrm{H}$ and their replacement by the quantity $\mathrm{U}$ plausibly concurred to their disappearance from the thermodynamic syntax.

\subsection{Clausius' Second Law for Cyclic Processes}

Clausius took almost 15 years to attain an accomplished mathematical expression of the Second Law. This long process went through a number of subsequent steps that reflect the evolution of Clausius' thought. At first, Clausius dealt with cyclic transformations; he subsequently provided the "extension of his second fundamental theorem" to non-cyclic processes [22].

Cyclic transformations were investigated within a logical framework grounded on the idea of uncompensated transformation [23] and the concept of Aequivalenzwerth [24]. The meaning and the relevance of these foundational elements have been thoroughly analyzed by other authors [13] and will not be further commented here. The historical evolution of Clausius' treatment of cyclic processes is well outlined in his 1872 paper, wherein he recognized two basic steps, dated 1854 and 1862, respectively.

In 1854, Clausius defined the quantity N (i.e., uncompensated transformation) and stated that it can only be positive or null: 
"In a treatise published in 1854 [25] I defined, for a given cyclic process, the quantity $\mathrm{N}$ that I named uncompensated transformation and it is defined by the equality:

$$
N=\int \frac{d Q}{T}
$$

where dQ is the heat element that is exchanged between the transforming system and the heat reservoir. $\mathrm{T}$ consequently represents the absolute Temperature. About this quantity [i.e., $\mathrm{N}], \mathrm{I}$ have demonstrated that it can only be positive, or at least null at the boundary condition represented by a reversible cyclic process." [7] (p. 144) (translated by the authors)

Eight years later, in 1862, he accomplished the mathematical translation of the foregoing statement by introducing the following well-known inequality:

"In a later treatise [26] I have further completed the analysis. The previous expression for the uncompensated transformation concerning only cyclic processes-leaving out the symbol $\mathrm{N}$ - can also be written as follows:

$$
\int \frac{d Q}{T} \geq 0
$$

that holds only for a cyclic process." [7] (p. 145) (translated by the authors)

\subsection{Clausius' Second Law for Non-cyclic Processes}

The formalization of the Second Law for cyclic processes relied on two macroscopic quantities with a well-defined physical meaning: heat and temperature. This approach was no longer possible for non-cyclic transformations. The treatment of such systems required Clausius to refer to a microscopic model, founded on two formal quantities: the heat actually contained in the body $(\mathrm{H})$ and the disgregation $(\mathrm{Z})$ [27]. Clausius briefed the meaning of disgregation in this remarkable paragraph that highlights its microscopic character:

"By disgregation is represented [...] the degree of dispersion of the body. Thus, for example, the disgregation of a body is greater in the liquid state than in the solid, and greater in the aeriform than in the liquid state. Further, if part of a given quantity of matter is solid and the rest liquid, the digregation is greater the greater the proportion of the whole mass that is liquid; and similarly, if one part is liquid and the remainder aeriform, the disgregation is greater the larger the aeriform portion. The disgregation of a body is fully determined when the arrangement of its constituent particles is given; but, on the other hand, we cannot say conversely that the arrangement of the constituent particles is determined when the magnitude of the disgregation is known. It might, for example, happen that the disgregation of a given quantity of matter should be the same when one part was solid and one part aeriform, as when the whole mass is liquid." [6] (p. 226)

Clearly, disgregation is neither a macroscopic physical quantity nor is it experimentally accessible. Its fuzzy character exposed it to several criticisms that will be tackled in the discussion section.

The mathematical expression of disgregation was first obtained for non-cyclic reversible changes. Under these conditions, Clausius postulated the relation between disgregation, heat and work, stating 
that - at a given temperature - the disgregation was "proportional to the work that the heat can thereby perform" and "the corresponding work must be proportional to the absolute temperature" [6] (p. 227). Here Clausius employs the term "work" to designate the total work (L), i.e., the sum of internal (I) and external (W) work. Clausius pointed out this relation as follows:

"Since the increase of disgregation is the action by means of which heat performs work, it follows that the quantity of work must bear a definite ratio to the quantity by which the disgregation is increased; we will therefore fix the still arbitrary determination of the magnitude of disgregation so that, at any given temperature, the increase of disgregation shall be proportional to the work which the heat can thereby perform. The influence of the temperature is determined by the foregoing law. For, if the same change of disgregation takes place at different temperatures, the corresponding work must be proportional to the absolute temperature." [6] (p. 227)

The formal translation of this statement is the following equation:

$$
d L=K T d Z
$$

where $\mathrm{K}$ is a constant, dependent on the measure unit of $\mathrm{Z}$, that can be chosen so that $K=\frac{1}{A}$, where $\mathrm{A}$ is the thermal equivalent of a unit of work. By simple substitution, Equation (7) becomes

$$
d L=\frac{T}{A} d Z
$$

In the case of non-cyclic irreversible changes, Clausius modified the relation between work, temperature and disgregation as follows:

"In case this last condition [i.e., reversible condition] need not be fulfilled, the change of disgregation may be greater, provided it is positive, than the value calculated from the work [i.e., total work, L]; and if negative, it may be, when taken absolutely, smaller than that value, but in this case also it would algebraically have to be stated as greater." [6] (p. 245)

The formal implication of this statement is the following inequality:

$$
d Z \geq \frac{A}{T} d L
$$

or equivalently

$$
d L \leq \frac{T}{A} d Z
$$

Through the substitution of $d L$ - given by the inequality (10) — in the First Law, formalized by Equation (4), Clausius obtained:

$$
d Q+d H+A \frac{T}{A} d Z \geq 0
$$

that could be rearranged as follows:

$$
\frac{d Q+d H}{T}+d Z \geq 0
$$

Equation (12) is the mathematical expression of the Second Law of the mechanical theory of heat for 
non-cyclic changes or-quoting the title of the Sixth Memoir-"The extension of the second fundamental theorem". This result constitutes the end of the story of the Second Law that Clausius remarkably summarized using these words:

"I wanted to obtain a quantity that was valid for any transformation of a system and whose value could change only in one sense. For this scope, in addition to both the already existing statements about transformation (i.e., the one concerning the transformation of work into heat and vice versa, and the one about the transfer of heat from a warmer system to a cooler and vice versa), I added a third one that regards the state change of a system and was formulated by means of a quantity Z, that I named the Disgregation of the system. With the aid of this quantity and of that indicated with $\mathrm{H}$, the heat actually available in the system, instead of the above relation I could obtain the following more general instance:

$$
\int \frac{d Q+d H}{T}+\int d Z \geq 0
$$

The sum:

$$
\int \frac{d H}{T}+\int d Z
$$

is that to which I have given the name of Entropy of a system.” [7] (pp. 145-146) (translated by the authors)

It is worth underlining that the definition of entropy stands as the ultimate step of the evolution of the Second Law. Definitely, entropy was not necessary for formulating the Second Law and its definitiongiven in 1865 [28] — can be contextualized in the attempt of Clausius to render the fundamental equations of the mechanical theory of heat [29] "more convenient for use", as he declared in the incipit of his Ninth Memoir [6] (p. 327). In fact, Equation (13) contains two quantities, the disgregation $\mathrm{Z}$ and the heat actually contained in the body $\mathrm{H}$, that have no macroscopic physical meaning and stems from Clausius' microscopic model. These quantities are not experimentally accessible and Equation (13) is unsuitable for practical applications. Clausius was well aware of that: in fact, he pointed out that, in the community of physicists and engineers (i.e., the final users of his fundamental equations of the heat theory):

“... difficulties of calculations are frequently encountered which retard progress and occasion errors. Under these circumstances I believe I shall render a service to physicists and mechanicians by bringing the fundamental equations of the mechanical theory of heat from their most general forms to others which, corresponding to special suppositions and being susceptible of direct application to different particular cases, are accordingly more convenient for use." [6] (p. 327)

Readily the definition of entropy implied a substantial formal simplification of the Second Law, allowing Clausius to abandon the disgregation $\mathrm{Z}$ and the thermal content of the body $\mathrm{H}$, and to formalize the Second Law in the usually known form [30]. Indeed, by substituting the entropy definition

$$
d S=\frac{d H}{T}+d Z
$$

into Clausius inequality 


$$
\frac{d Q+d H}{T}+d Z \geq 0
$$

Equation (12) transforms as follows:

$$
\frac{d Q}{T}+d S \geq 0
$$

Rearranging the inequality and changing the sign of heat — in order to adopt the convention that the sign is positive when heat is absorbed by the system [31] - the Second Law for non-cyclic processes becomes:

$$
d S \geq \frac{d Q}{T}
$$

where the equality holds exclusively in the case of reversibility. When the system is adiabatically isolated (i.e., $d Q=0)$, inequality (18) becomes:

$$
\mathrm{d} S \geq 0
$$

In summary, the first formal expression of Clausius' Law for non-cyclic processes — dating 1862 — was given in terms of disgregation $(\mathrm{dZ})$, heat actually contained in the body $(\mathrm{dH})$ and heat exchanged with the environment $(d Q)$. It is only in 1865 that Clausius provided the definition of a new quantity, called entropy (S), defined as in Equation (15), with the explicit purpose to render the fundamental equations of the mechanical theory of heat "more convenient for use" [6] (p. 327), i.e., for simplifying the mathematical expression of the Second Law and make it exploitable in practical contexts. The effectiveness of this choice is evident from Table 1 that reports a comparison of the expressions of the Second Law for non-cyclic thermodynamic changes.

Table 1. Comparison of the expressions of the Second Law for non-cyclic thermodynamic changes.

\begin{tabular}{|l|c|c|}
\hline \multicolumn{1}{|c|}{ Type of transformation } & $\begin{array}{c}\text { Formulation of the } \\
\text { Second Law in 1862 }\end{array}$ & $\begin{array}{c}\text { Formulation of the } \\
\text { Second Law in 1865 }\end{array}$ \\
\hline Any non-cyclic transformation & $\frac{d H+d Q}{T}+d Z \geq 0$ & $d S \geq \frac{d Q}{T}$ \\
\hline Reversible non-cyclic transformation & $\frac{d H+d Q}{T}+d Z=0$ & $d S=\frac{d Q}{T}$ \\
\hline
\end{tabular}

\section{Discussion}

The results of the foregoing epistemological analysis are schematically summarized in the flow chart reported in Figure 1, wherein three main logical steps are identified. These steps show that:

1) The 1862 formulation of the Second Law is the result of the introduction of inequality $d L \leq \frac{T}{A} d Z$ into the mathematical expression of the First Law. This step conceals two critical points: the postulation of the value of disgregation (related to total work and temperature) as well as its formal substitution into the equation that expresses the principle of conservation of energy. Sands and Dunning-Davies [13] as well as Meixner [32] have made serious remarks to this operation, as it would affect the consistency between First and Second Law in the case of irreversible processes. For a detailed 
discussion of these aspects the reader should refer to these authors' works, as this problem is beyond the scope of the present paper. We would just like to underline that, besides the inconsistencies that might be found in Clausius' formal system, Clausius' work exhibited an unquestionable conceptual fertility and paved the way for all subsequent developments of modern thermodynamics.

2) In 1865 Clausius provided the definition of a new quantity, called entropy (S), defined as in Equation (15), with the aim of simplifying the formulation of the Second Law and make it exploitable in practical contexts. The final result was the reformulation of the Second Law as the well-known inequality (18).

3) When a system is adiabatically isolated (i.e., $d Q=0$ ) the expression of the Second Law becomes $d S \geq 0$; this inequality (that will not be further discussed in the present paper) is the mathematical form of the well-known statement that the entropy of an adiabatically isolated system tends to a maximum.

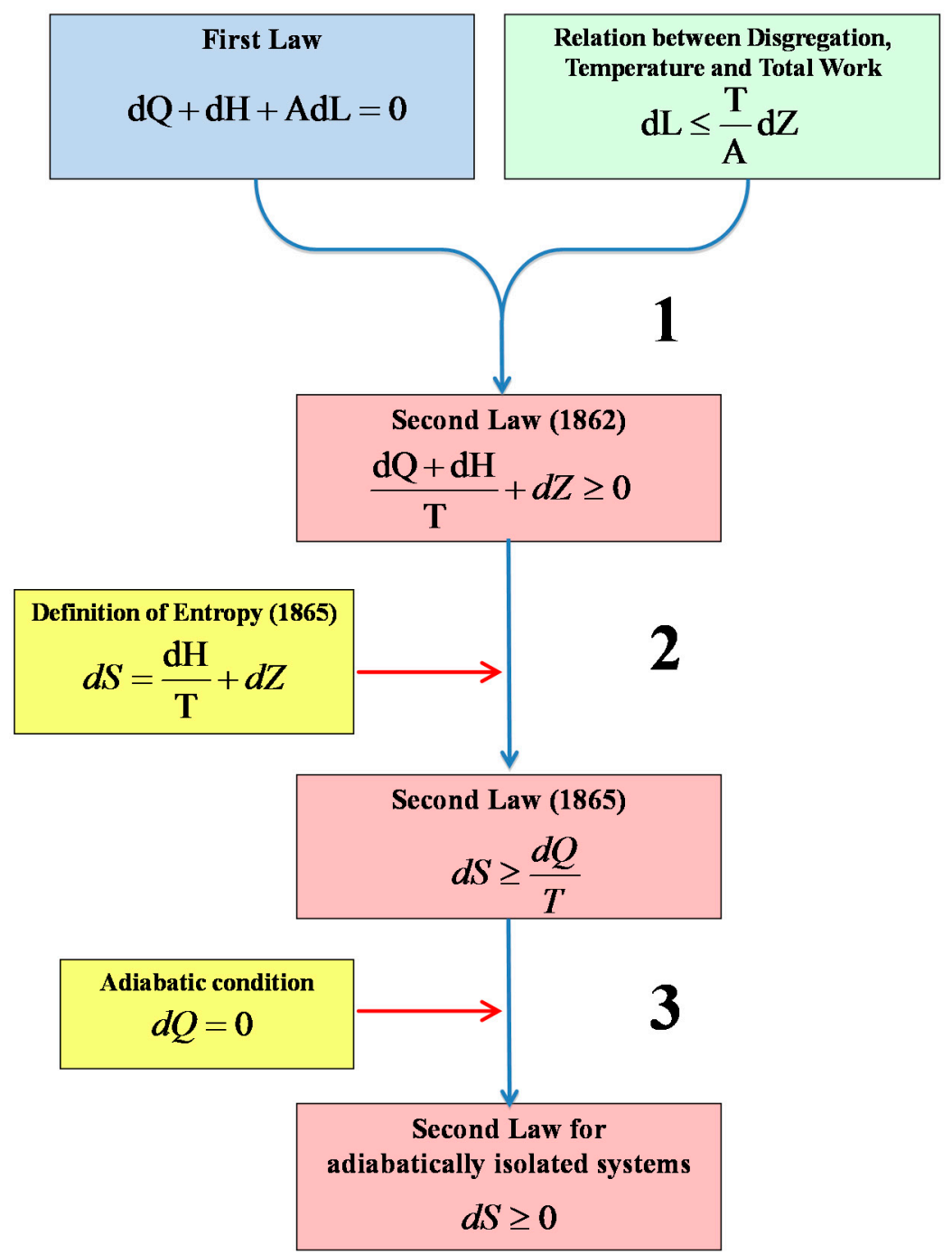

Figure 1. Schematic representation of the pathway that brought to the formulation of the Second Law.

Our analysis led us to focus on three conceptual aspects that will be discussed in detail: 
i) The formal expression of the Second Law - as it is known today - is the result of the extension of Clausius' second fundamental theorem to non-cyclic transformations. Clausius' logical process is not grounded on entropy but rather on a concept that has now disappeared from the thermodynamic language: the disgregation.

ii) Despite its disappearance and its apparent oddity, disgregation has an epistemic relevance, as it discloses the cognitive strategy chosen by Clausius to tackle the difficult problem of non-cyclic transformations.

iii) The disappearance from the thermodynamic horizon was the common fate of disgregation and other pre-modern quantities. Some hypotheses on the reasons for such disappearance are discussed.

\subsection{The Relationship between Entropy and the Second Law}

The first formal expression of Clausius' Law_dating 1862 - does not contain entropy at all. In 1862 the Second Law was rather formalized in terms of the quantities: disgregation $(d Z)$, heat exchanged with external bodies $(d Q)$ and heat actually contained in the body $(d H)$. Entropy entered the formalization of the Second Law only three years later. The first definition of entropy is found in Clausius' Sixth Memoir and is given in terms of $\mathrm{H}$ and $\mathrm{Z}$, according to Equation (15). This syntactic move simplifies the expression of the Second Law and, according to Clausius' words, specifies "the physical meaning of the magnitude S" [6] (p. 355) within his microscopic model. Nevertheless, Equation (15) is not useful for calculations. It is only in his Ninth Memoir that Clausius provides "a way for determining S" for reversible processes. By integrating the expression

$$
d S=\frac{d Q}{T}
$$

that is Clausius' Second Law for reversible processes - he obtained the following operational definition of entropy:

$$
S=S_{0}+\int \frac{d Q}{T}
$$

where $S_{0}$ is the value which the magnitude $\mathrm{S}$ has in the initial conditions [6] (pp. 354-355).

Clausius did not provide any exploitable definition of entropy for irreversible transformations and the treatment of these processes is still matter of debate.

\subsection{Epistemic Relevance of Disgregation}

Our analysis shows that the introduction of the concept of disgregation stands out as a mandatory step on the pathway that led Clausius to the formalization of the Second Law for non-cyclic thermodynamic systems. Faced with the problem of non-cyclic transformations, Clausius realized the impossibility of relying exclusively on the macroscopic quantities work and heat. A new, original strategy was needed: Clausius chose to refer to a microscopic model for representing thermodynamic systems and to use a new quantity, disgregation, for describing the state of the system in terms of particles' dispersion. These premises allowed him to achieve a first mathematical expression of the 
Second Law for non-cyclic systems (1862). This step would have not been possible without relying on the idea of disgregation.

As disgregation stems from a microscopic model, the fact that it played a crucial role in the treatment of non-cyclic processes is further evidence that a microscopic view of thermodynamic systems was in Clausius' mind when he developed his theory of heat up to the formulation of the Second Law.

The scientists' opinions on the meaning and relevance of $Z$ are not unanimous. Some of them consider that its lack of macroscopic physical meaning and its disappearance from the thermodynamic language make this quantity an irrelevant element that, in addition, can be a source of deep contradictions. Indeed, the meaning of disgregation seems so distant from the current view that some authors look at it as an "abstruse" notion. This is the case of Uffink:

"A further paper (Clausius 1862) presents what in his collected work is referred to as the 'extended form' of the Second Law. Here, he studies processes where the final state of the system differs from the initial state. For convenience I will call these 'open processes'. For this purpose Clausius needs a number of assumptions about the possible change of states of that system, and hence about its internal constitution. He characterizes the state of the system by introducing two abstruse quantities: the 'vorhandene Wärme' $\mathrm{H}$ and the 'Disgregation' Z. The definition of these quantities is not very clear (Clausius merely remarks about the Disgregation that it represents a 'degree of distribution', which is related to the ordering of the molecules) and for our purpose actually not very important.” [5] (p. 29)

A different opinion is expressed by Klein, who points out that:

"Clausius saw the disgregation as a concept more fundamental than the entropy, since entropy was to be interpreted physically with the help of disgregation. He found entropy most useful as a summarizing concept. This viewpoint comes out particularly clearly in a lecture Clausius gave in 1867 on the Second Law to the German Scientific Association. He explained and analyzed several aspects of the law in considerable detail, with disgregation playing an important part in the discussion. It is only in the concluding paragraphs of his talk that Clausius brought in entropy at all, to pull the ideas together.” [33] (p. 140)

Interestingly, many scientists who were Clausius' contemporaries were favorable towards disgregation. This was the case of Gibbs, who after discussing the microscopic meaning of disgregation and its relationship with temperature and work, comments:

"Although they were advanced rather as a hypothesis than as anything of which he could give a formal proof, he seems to have little doubt of their correctness, and his confidence seems to have increased with the course of time. The substantial correctness of these views cannot now be called in question. The researches especially of Maxwell and Boltzmann have shown that the molecular vis viva is proportional to the absolute temperature and Boltzmann has been able to determine the precise nature of the functions which Clausius called entropy and disgregation." [34] (p. 264)

Remarkably, in the same writing, Gibbs states that Clausius regarded disgregation as "determined by 
the positions of the elementary parts of the body without reference to their velocities" [34] (p. 264). Klein remarks that this is Gibbs' interpretation:

"As a matter of fact, Clausius had never discussed this point [i.e., the velocity of particles] at any length. He had repeatedly said that the disgregation was fully determined by the arrangement of the constituent molecules of the body, but he had not emphasized that it was not determined by molecular velocities.” [33] (p.143)

These few lines highlight that disgregation is a key-concept within the atomic-molecular models of thermodynamics systems as it stands as a cognitive tool bridging the macroscopic and microscopic levels. It may be seen as a proto-function, playing a role that - in the 20th century's thermodynamics and statistical mechanics — would be accomplished by entropy. Despite its conceptual incompleteness, disgregation was fertile in paving the way for intuitions and subsequent developments of thermodynamic theories based on Clausius' premises.

A further element that shows the relevance of disgregation within the thermodynamic community in the second half of the 19th century is highlighted by Kragh and Weininger that report Horstmann's position:

"Although Horstmann explicitly used entropy as an important chemical quantity, he based much of his discussion on the notion of disgregation, which Clausius had introduced in 1862 as a measure of the separation of parts of a system from one another. Disgregation does not occur in later textbooks in thermodynamics but in the 1860s it played an important role not only as an immature version of entropy, but also in its own right. In his work of 1862, Clausius argued that the notion of disgregation would be particularly useful in chemistry as a measure of affinity, and it was this idea that Horstmann developed.” [35] (p. 97)

Indeed, Clausius himself recognized the high potential of disgregation as a cognitive tool and foresaw it as a useful element for extending the Second Law to chemical systems:

"I believe, indeed, that we must extend the application of this law, supposing it to be correct, still further, and especially to chemical combinations and decompositions. The separation of chemically combined substances is likewise an increase of the disgregation, and the chemical combination of previously isolated substances is a diminution of their disgregation; and consequently these processes may be brought under considerations of the same class as the formation or precipitation of vapor. That in this case also the effect of heat is to increase the disgregation, results from many well-known phenomena, many compounds being decomposable by heat into their constituents — as, for example, mercuric oxide, and, at very high temperatures, even water." [6] (p. 240)

\subsection{Reasons for the Disappearance of Disgregation}

The fact remains that, despite being used by the scientific community in the 1860 s, the concept of disgregation faded out and finally disappeared from the thermodynamic language, to the point that today there are no traces left of it. A similar fate occurred to the thermal content $\mathrm{H}$ and the internal work I. What are the reasons for such event? 
Although a straightforward answer is difficult to be stated, some reasonable hypothesis may be formulated. We have sought for the features shared by these three pre-modern quantities. All of them belong to the microscopic model whereon Clausius' formal system is grounded. Namely, they are not measurable physical quantities but microscopic formal parameters whose raison d'être lies in their belonging to a specific formal system, i.e., Clausius' microscopic model. As long as this latter held, premodern functions could be part of the thermodynamic syntax. Since the end of the 19th century, Clausius' microscopic idea of matter was overridden by new, more effective models. Nowadays it stands as an archaic model, no longer valid. Consequently, parameters such as disgregation, $\mathrm{H}$ and $\mathrm{I}$ - intimately attached to this ancient microscopic view of matter-have not survived.

A further reason, suggested by Leff, is that "Clausius' disgregation-based theory led him to an incorrect result on specific heats; so Clausius decided to delete disgregation from the second edition of his book on the mechanical theory of heat" [36] (p. 1270).

In our opinion, these overall reasons may justify the disappearance of the three "pre-modern" thermodynamic functions $\mathrm{H}, \mathrm{I}$, and $\mathrm{Z}$ from modern thermodynamics.

\section{Conclusions}

Disgregation, as well as the heat actually contained in the body and the internal work, are no longer present in the thermodynamic vocabulary and some authors see them as conceptual relics of no interest in the modern landscape of thermodynamic studies.

Our analysis shows that disgregation is a crucial logical step of the pathway that led Clausius towards the translation of the Second Law into mathematical expressions. These pre-modern quantities were essential for building up Clausius' formal system. Their role may be effectively represented through a metaphor borrowed from the language of architecture: they may be seen as the construction lines traced to build up the thermodynamic formal apparatus. They could finally vanish from the formal description of thermodynamic systems without compromising its conceptual self-consistency. In fact, the final result of Clausius' treatment, i.e. $d S \geq \frac{d Q}{T}$, still holds without relying any longer on Clausius' archaic microscopic model.

The epistemic relevance of disgregation has been pointed out by outstanding scientists, such as Gibbs and Horstmann. We add further elements by showing that Clausius relied on this concept to formalize the Second Law for non-cyclic systems. Further, in agreement with Leff [36], we show that disgregation, intended as a measure of particles' dispersion, helps to clarify the meaning of entropy in Clausius' thought. In this perspective, pre-modern quantities shed light on the comprehension of the Second Law.

\section{Acknowledgments}

We thank the anonymous reviewers whose suggestions allowed a significant improvement of the quality of the present work. 


\section{Author Contributions}

Emilio Marco Pellegrino and Elena Ghibaudi conceived the paper's architecture and wrote the paper. Luigi Cerruti gave a substantial contribution to the discussion. All authors have read and approved the final manuscript.

\section{Conflicts of Interest}

The authors declare no conflict of interest.

\section{References and notes}

1. Č́ápek, V.; Sheehan, D.P. Challenges to the Second Law of Thermodynamics; Springer: Dordrecht, The Netherlands, 2005.

2. Nikulov, A.; Sheehan, D. The Second Law Mystique. Entropy 2004, 6, 1-10.

3. Larmor, J.; Bryan, G.H. On the Present State of our Knowledge of Thermodynamics, Especially with Regard to the Second Law. In Report of the Sixty-first Meeting held at Cardiff in August 1891; British Association for the Advancement of Science, Ed.; John Murray: London, UK, 1891; pp. 85122.

4. Bridgman, P.W. The Nature of Thermodynamics; Harvard University Press: Cambridge, MA, USA, 1941.

5. Uffink, J. Bluff Your Way in the Second Law of Thermodynamics. Stud. Hist. Philos. Sci. B 2001, 32, 305-394.

6. Clausius, R.; Hirst, T.A. The Mechanical Theory of Heat: With Its Application to the Steam Engine and to the Physical Properties of Bodies; J. Van Voorst: London, UK, 1867.

7. Clausius, R. Zur Geschichte der Mechanischen Wärmetheorie. Annalen der Physik und Chemie 1872, $145,132-146$. This paper has been translated by the authors of the present manuscript, as there is no English version available of it.

8. Müller, I. A History of Thermodynamics: The Doctrine of Energy and Entropy; Springer: Berlin, Heidelberg, Germany, 2007. Concerning Clausius' disputes with the scientific community, Müller reports that "in his work on thermodynamics he had to fight off numerous objections and claims of priority by other people, who had thought, or said, or written something similar at about the same time".

9. Müller reports that the Newcomen's steam engine was actually developed by the engineer Thomas Savery (1650-1715) and the blacksmith Thomas Newcomen (1663-1729). Newcomen's machine worked cyclically by injection of cold water into the cylinder where the steam was condensed and a good vacuum was developed. The vacuum pulled down the piston in a powerful stroke and subsequently new steam pushed the piston back up. At this stage a new cycle began by injecting cold water into the cylinder. See [8] (p. 48).

10. As regards the efficiency of the Newcomen's steam engine, Müller reports: "Actually, the efficiencies were all quite low: in Newcomen's case about $2 \%$ and 5-7\% in Watt's case. A modern power station reaches between $45 \%$ and $50 \%$. The engineers have done a good job indeed over the past 200 years" [8] (Footnote in p. 50). 
11. Watt's major invention was the rotative engine-where the up-and-down movement of the piston is converted into the rotation of a wheel. It opened to the use of steam machines as driving force for ships and locomotives. On this matter Müller reports: "Thus Watt's machine became the motor of the industrial revolution" [8] (p. 49).

12. Carnot, S. Reflections on the Motive Power of Heat, 2nd ed.; Wiley: Hoboken, NJ, USA, 1897.

13. Sands, D.; Dunning-Davies, J. Clausius' Concepts of "Aequivalenzwerth" and Entropy: A Critical Appraisal. In The Physics of Reality: Space, Time, Matter, Cosmos-Proceedings of the 8th Symposium Honoring Mathematical Physicist Jean-Pierre Vigier; Amoroso, R.L., Kauffman, L.H., Rowlands, P., Eds.; World Scientific: Singapore, Singapore, 2013; pp. 207-217.

14. Müller underlines the relevance of Carnot's work for thermodynamics and writes: "Had he lived longer, it seems likely that he might have anticipated Clausius' work by nearly 30 years" [8] (p. 55).

15. Clausius, R. Über die Art der Bewegung, Welche Wir Wärme Nennen. Annalen der Physik und Chemie 1857, 176, 353-380.

16. Brush, S.G. The Kind of Motion We Call Heat: A History of the Kinetic Theory of Gases in the 19th Century; North-Holland: Amsterdam, The Netherlands, 1976.

17. Clausius, R. The Nature of the Motion which we Call Heat. In Kinetic Theory (The Commonwealth and International Library: Selected Readings in Physics); Brush, S.G., Ed.; Pergamon Press: Oxford, UK, 1965; pp. 111-134.

18. The convention is to take the sign of heat $(\mathrm{Q})$ positive when heat flows from the system towards the exterior. The German expression used for $\mathrm{H}$ is im Körper vorhandenen Wärme, translated as the heat actually contained in the body, in the English version of the Abhandlungen edited in 1867. Appendix A of the Sixth Memoir, consecrated to terminology, reports: "the heat actually present in the body may be simply called the heat of the body or the thermal content of the body (Wärmeinhalt des Körpers)" [6] (p. 252) [Italics in the text].

19. Equation (1) is reported in a footnote of Clausius' Sixth Memoir [6] (p. 225 footnote). Concerning the equivalence of heat and work, in Appendix A of the Sixth Memoir, Clausius writes: "Now in the mechanical theory of heat, after admitting that heat can be transformed into work and work into heat, in other words, that either of these may replace the other, it becomes frequently necessary to form a magnitude of which heat and work are constituent parts. But heat and work being measured by different units, we cannot in such a case say, simply, the magnitude is the sum of heat and work; we are compelled to say either the sum of the heat and the heat-equivalent of the work, or the sum of the work and the work-equivalent of the heat" [6] (pp. 252-253). Within this frame of ideas, Clausius proposed the introduction of a new quantity, the ergon, that is the work measured according to the heat units. He writes: "But beside the work measured according to mechanical unit, let another magnitude be introduced denoting the work measured according to the thermal unit, that is to say, the numerical value of the work when the unit of work is that which is equivalent to the thermal unit. For the work thus expressed a particular name is requisite. I propose to adopt for it the Greek word (Épyov) ergon [6] (pp. 252-253)" [Italics in the text].

20. The denomination "energy" for the quantity $\mathrm{U}$ is due to $\mathrm{W}$. Thomson, as Clausius himself reports: "Later, Thomson gave to this function - designated by me with U- the very appropriate name of energy of the body" [7] (p. 136) (translated by the authors). It is worth reporting the justification given by Clausius for having defined the function $U$ [6] (p. 225, footnote): "Since, however, the 
increase in the quantity of heat actually contained in a body $[\mathrm{dH}]$, and the heat consumed by an interior work during a change of condition $[A d I]$, are magnitudes of which we commonly do not know the individual values, but only the sum of those values, and which resemble each other in being fully determined as soon as we know the initial and final conditions of the body, without our requiring to know how it has passed from the one to the other, I have thought it advisable to introduce a function which shall represent the sum of these quantities and which I have denoted by U. Accordingly:

$$
d U=d H+A d I
$$

21. Knott, C.G. Life and Scientific Work of Peter Guthrie Tait Supplementing the Two Volumes of Scientific Papers Published in 1898 and 1900; Cambridge University Press: Cambridge, UK, 1911; p. 222.

22. The sentence is quoted as it corresponds to the title of Clausius Sixth Memoir [6] (pp. 215-266).

23. Clausius clarified his idea of compensation in a footnote of his Fourth Memoir that we quote here below: "Now it is to these compensations that our principle [i.e., Clausius' Second Law] refers; and with the aid of this conception the principle may also be expressed thus: an uncompensated transmission of heat from a colder to a warmer body can never occur. The term 'uncompensated' here expresses the same idea as that which was intended to be conveyed by the words 'by itself' in the previous enunciation of the principle [see Clausius' statement of the Second Law at p. 4]" [6] (p. 118, footnote).

24. Sands and Dunning-Davies remark: "Mathematically, Clausius treated compensation through the concept of Aequivalenzwerth or equivalence value, which was a quantity with the value $Q / T, Q$ being a quantity of heat transmitted at a temperature T" [13] (p. 5).

25. Clausius, R. Annalen der Physik und Chemie; 1854, 113, 499. This issue is also discussed in Clausius' Fourth Memoir [6] (pp. 111-135).

26. Clausius, R. Annalen der Physik und Chemie; 1862, 116, 73. This issue is also discussed in Clausius' Fourth Memoir [6] (pp. 111-135).

27. The extension of the Second Law to non-cyclic processes is treated in Clausius' Sixth Memoir [6] (pp. 215-266).

28. The definition of entropy is reported in the Ninth Memoir, On Several Convenient Forms of the Fundamental Equations of the Mechanical Theory of Heat. According to the footnote reported in its incipit, this memoir was the object of a lecture "read at the Philosophical Society of Zurich on the 24th of April 1865 and published in the Vierteljahrsschrift of this Society, Bd. X. S. 1.; Pogg. Ann. July, 1865, Bd. Cxxv. S. 353; Jour. De Liouville, $2^{\circ}$ sér. T. x. P. 361” [6] (p. 327).

29. The fundamental equations correspond to the analytical expressions of the two fundamental theorems whereon the whole mechanical theory of heat rests: "that of the equivalence of heat and work and that of the equivalence of transformations" [6] (p. 327).

30. The ultimate implication of the definition of Entropy was the object of the Appendix to the Ninth Memoir published in 1866-whose aim was to "show how the equations which serve for the determination of energy and entropy may be derived directly from the fundamental equations of the mechanical theory of heat". In this context Clausius could "bring the two equations [i.e., $\mathrm{dQ}=\mathrm{dU}+\mathrm{dw}$ and $\mathrm{dQ}=\mathrm{TdS}$ ] into conjunction"- - under the supposition of reversible change of a body [6] (p. 366). It is remarkable that the result attained has the form of what today is known 
as the "first Gibbs equation" and that would have constituted the foundational element of Gibbs thermodynamics [34] (pp. 33-54). It is worth to quote Clausius on this particular matter: "In this case [i.e., reversible change] the thermal element dQ is the same in both equations, hence we can eliminate it from the equations, whereby we obtain $\mathrm{TdS}=\mathrm{dU}+\mathrm{dw}$ " [6] (p. 366).

31. Clausius assumed the opposite convention; in fact, as regard to the heat element, he specified that "dQ is the heat element that flows from the body under transformation towards a heat reservoir" [7] (p. 144) (translated by the authors).

32. Meixner, J. Processes in Simple Thermodynamic Materials. Arch. Ration. Mech. Anal. 1969, 33, $3-53$.

33. Klein, M.J. Gibbs on Clausius. Hist. Stud. Phys. Sci. 1969, 1, 127-149.

34. Gibbs, J.W. The Scientific Papers of J. Willard Gibbs; Bumstead, H.A., van Name, R.G., Eds.; Longmans: London, UK, 1906.

35. Kragh, H.; Weininger, S.J. Sooner Silence than Confusion: The Tortuous Entry of Entropy into Chemistry. Hist. Stud. Phys. Biol. Sci. 1996, 27, 91-130.

36. Leff, H.S. Thermodynamic Entropy: The Spreading and Sharing of Energy. Am. J. Phys. 1996, 64, $1261-1271$.

(C) 2015 by the authors; licensee MDPI, Basel, Switzerland. This article is an open access article distributed under the terms and conditions of the Creative Commons Attribution license (http://creativecommons.org/licenses/by/4.0/). 